\title{
Descripción de los complejos estructurales óseos en Helcogrammoides chilensis (Blennioidei: Tripterygiidae) de la zona central de Chile
}

Description of the bony structure complexes in Helcogrammoides chilensis

(Blennioidei: Tripterygiidae) of the central zone of Chile

\section{Cecilia Cancino', 2 , Katerin Farías ${ }^{3}$, Sabrina Lampas ${ }^{3}$, Betzabé González ${ }^{3}$ y Victoria Cuevas}

\begin{abstract}
${ }^{1}$ Escuela de Recursos Naturales, Universidad del Mar, Angamos 680, Reñaca, Viña del Mar, Chile. cecilia.cancino@udelmar.cl ${ }^{2}$ Departamento de Ecología y Biodiversidad, Facultad de Ecología y Recursos Naturales, Universidad Andrés Bello, República 470, Santiago, Chile

${ }^{3}$ Centro Innovamar, Universidad del Mar, Amunátegui 1838, Recreo, Viña del Mar
\end{abstract}

\begin{abstract}
This paper describes the osteological features in the context of the structural complexes they build as a way of avoiding their separation from the morphofunctional wholes to which they belong. The posterior zone of the neurocranium is compact, and the splanchnocranium is laminar with very little cartilaginous tissue sustaining robust oral jaws. The pharyngobranchial apparatus is short anteroposteriorly and expanded laterally. The hyoid bar is small, about as long as its branchiostegal rays. The spatial relationship of the paired and unpaired fins to the vertebral column was highly constant in the specimens examined of Helcogrammoides chilensis from the Central zone of Chile. The absence of a neural spine in the first two vertebrae, a highly fused caudal complex, the urohyal with well developed lateral processes, and presence of a pelvic spine, are osteological features that contribute to the diagnosis of Helcogrammoides.
\end{abstract}

Key words: Morphology, osteology, triplefin blenny

\begin{abstract}
Resumen.- El presente trabajo plantea la descripción de estructuras osteológicas en función de los complejos estructurales que conforman, evitando así la desagregación de las totalidades morfofuncionales. El neurocráneo es compacto en la mitad posterior y el esplancnocráneo es laminar con muy poco tej ido cartilaginoso que sostiene robustas piezas bucales. Aparato faringobranquial achatado anteroposteriormente y expandido en forma lateral. La barra hioídea es pequeña, cuya longitud es similar a la longitud de sus rayos branquiostegales. La relación espacial de las aletas pares e impares a la columna vertebral es altamente constante en los especímenes de Helcogrammoides chilensis, de la zona central de Chile. La ausencia de espina neural en las dos primeras vértebras, complejo uróforo altamente fusionado, urohial con procesos laterales desarrollados y presencia de espina pélvica son caracteres que contribuyen a la diagnosis de Helcogrammoides.
\end{abstract}

Palabras clave: Morfología, osteología, trambollito

\section{INTRODUCCIÓN}

La familia Tripterygiidae, ha sido objeto de numerosos estudios (Regan 1912, Rosenblatt 1960, Wheeler \& Dunne 1975, Clark 1979, Hadley 1986, Gon 1990, Bussing 1991, Fricke 1991, Springer 1993, Castillo \& Pequeño 1998) centrados en la descripción morfológica de sus especies, con el objeto de resolver los problemas taxonómicos.

En Chile, se ha reconocido la presencia de tres especies de tripterígidos conocidos como trambollitos de tres aletas, debido a que la aleta dorsal es tripartita. Fowler en 1945 (vide in Castillo \& Pequeño 1998) reconoce la presencia de Tripterygion cunninghami Smitt, 1898; luego en 1955,
Cancino describe a Tripterygion chilensis, mientras que Tomo en 1981 describe a Tripterygion antarcticus.

Si bien estas especies fueron descritas por sus autores como pertenecientes al género Tripterygion Risso, 1826, Gon en 1990, al estudiar especímenes de T. cunninghami y T. antarcticus, valida la propuesta de Rosenblatt (1959), al ratificar los caracteres «ausencia de espina en la aleta anal» y «presencia de línea lateral continua y completa» como caracteres propios del género Helcogrammoides Rosenblatt, 1990. Por esta razón, Castillo \& Pequeño (1998) afirman que el único género de tripterígido presente en Chile 
es Helcogrammoides y establecen los rangos de distribución para cada una de las especies continentales, ubicando a Helcogrammoides cunninghami Smitt, 1898, entre Coquimbo y Tres Montes, mientras que el rango de distribución para Helcogrammoides chilensis, Cancino fide De Buen 1960, lo define entre Antofagasta y Talcahuano. Tripterygion antarcticus es documentada solo para la isla Bryde, en la Península Antártica Chilena.

A pesar de estos avances, los problemas taxonómicos en la familia Tripterygiidae persisten, ya que al estar conformada por 31 géneros cuyas diagnosis se fundamentan en la descripción de caracteres externos y la mayoría merístico, se produce algún grado de solapamiento cuando se identifican los especímenes. Dicha situación es claramente observable al estudiar el taxón Helcogrammoides, el cual comparte caracteres con Tripterygion, Enneapterygius, Helcogramma, Ceratobregma, Lepidonectes y Roanoho, tales como la presencia de tres espinas en la primera aleta dorsal (Wheeler \& Dunne 1975, Clark 1979, Hadley 1986, Fricke 1991, Eschmeyer 2002). La ausencia de espina anal también es carácter de Cryptichthys, Lepidoblennius y Blennodon (Gon 1990). En Cryptichthys, Blennodon y Enneanectes (Gon 1990) la línea lateral es continua y con escamas tubulares.

Es por ello que Regan (1912) es el primero en incorporar caracteres osteológicos en la diagnosis en peces, sin embargo el problema de identificación no se resuelve cuando ellos son tratados de manera independiente. Posteriormente Starks (1926), Weitzman (1962), Quast (1965), Dullemeijer \& Barel (1977), Barel (1983), Bock \& Zander, (1986) y Kotrschal (1988) plantean que el valor de los caracteres osteológicos radica en la posibilidad de determinar «complejos estructurales» (sensu Wiley 1981) que al ser delimitados por las relaciones de continuidad espacial entre las estructuras (Dullemeijer 1974, Lauder \& Liem 1983) definen las unidades funcionales que habilitan a los organismos a desempeñarse en su hábitat. Dichos complejos estructurales en peces han sido determinados por Liem (1970) a partir de análisis morfofuncionales.

Por lo anterior, el objetivo del presente trabajo consiste en describir en forma gráfica y explicativa la osteología de los complejos estructurales de especímenes de Helcogrammoides chilensis, para el establecimiento de un nuevo nivel de comparación estructural.

\section{MATERIAL Y MÉTODOS}

Se utilizaró un total de 15 ejemplares adultos de H. chilensis con longitudes estándar entre 45 y 65 mm, mantenidos en formalina al 10\%, recolectados por el Dr. Eduardo De la Hoz y la Prof. Cecilia Cancino en Valparaíso, entre los años 1994 y 1996. Además, se utilizaron dos especímenes donados por el Dr. Germán Pequeño para comparación. La identificación taxonómica de los especímenes se basó en el trabajo de Castillo \& Pequeño (1998).

Para la observación de las estructuras osteológicas se utilizaron doce especímenes diafanizados según técnica descrita por Dingerkus \& Uhler (1977) y las figuras fueron confeccionadas con un microscopio Wild M-7 provisto de cámara lúcida. Posteriormente, las figuras de la región cefálica fueron sombreadas y dibujadas con tinta por la Sra. Carmen Tobar, dibujante científico de la Facultad de Ciencias de la PUCV. Las áreas con mayor densidad de puntos pequeños corresponden a tejido óseo, mientras que las áreas con puntos de mayor tamaño indican zonas de tejido cartilaginoso.

Para la nomenclatura ósea se sigue a Cancino et al. (1999) y la lista de abreviaturas se muestra en el Anexo 1. Para la identificación y descripción de los complejos estructurales se sigue a Liem (1970). Se confeccionó una tabla de caracteres merísticos para la familia, basada en la revisión bibliográfica.

\section{RESULTADOS Y DISCUSIÓN}

Los resultados merísticos de los especímenes analizados en este trabajo concuerdan con aquellos descritos por Castillo \& Pequeño (1998) en la diagnosis del género Helcogrammoides, sin embargo estos autores no hacen mención a la presencia de una espina y un tercer radio en la aleta pélvica, presente en todos los especímenes analizados (Tabla 1). 

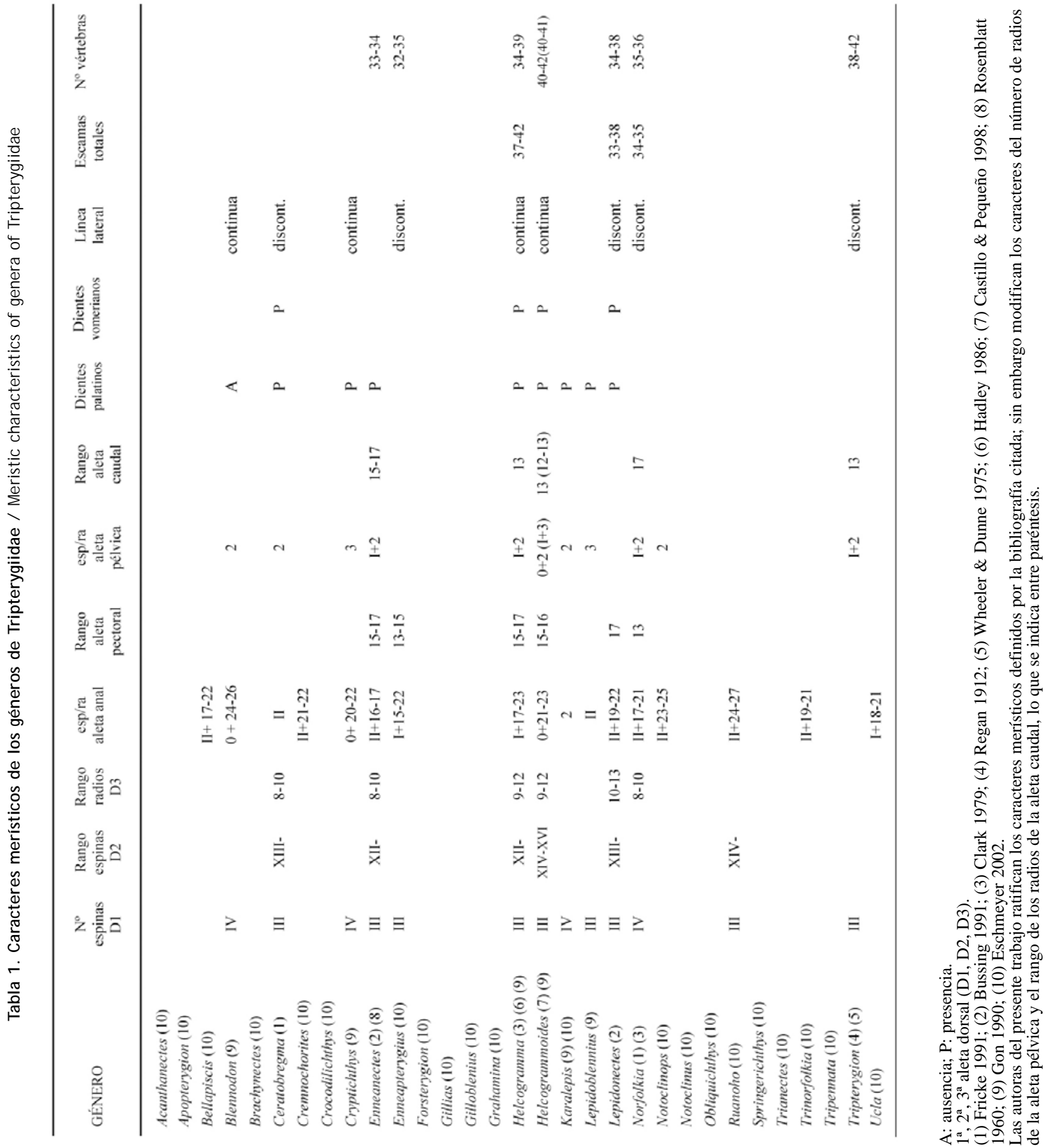


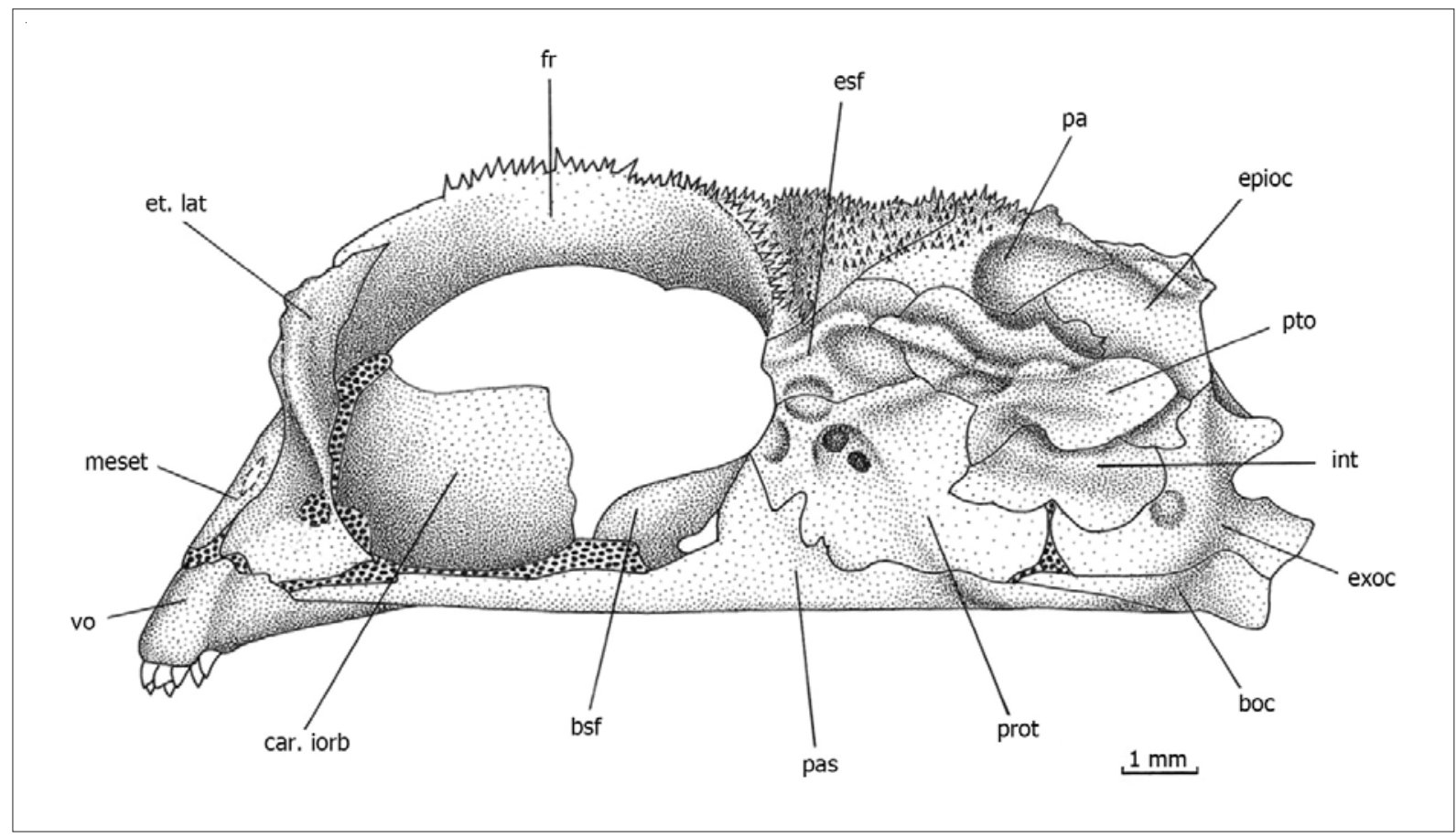

Figura 1. Vista lateral del neurocráneo de H. chilensis, los extraescapulares y el postemporal no están representados / Lateral view of the neurocranium of $\mathrm{H}$. chilensis, the extrascapular and postemporal bones were removed

\section{NEUROCRÁNEO}

El neurocráneo presenta escaso tejido cartilaginoso, en la mitad anterior se observa entre el vómer, mesetmoides, etmoides lateral y cartílago interorbital; este último se presenta mayoritariamente osificado, carácter que Hadley (1986) y Springer (1993) describen para Tripterygiidae. En la mitad posterior, el cartílago está restringido a la zona articular entre proótico, exoccipital y basioccipital (Fig. 1 y 3). Las articulaciones entre los huesos son de tipo sinartrosis, producto de la superposición de los bordes distales de las estructuras y presencia de cortas fibras colágenas que las mantienen firmemente unidas.

Se destaca la presencia de abundantes y pequeñas espinas sostenidas por el frontal y borde anterior del parietal (Fig. 1). Estas espinas también se presentan en el borde sagital del extraescapular y del post-temporal, las cuales no están representadas en las figuras.

El etmoides lateral es macizo, forma la pared anterior de la órbita ocular y se proyecta lateralmente originando el proceso preorbitario, con el cual articula al lacrimal; además, presenta una proyección anterior cóncava que aloja al nasal y donde se ubica el foramen olfatorio, carácter que es definido por Starks (1926) para osteíctios (Fig. 1). Ambos etmoides laterales se unen centralmente al vómer (Fig. 2).
El mesetmoides impar se une firmemente a los etmoides laterales por medio de tejido conectivo. Anteriormente limita con el vómer, mientras que el borde se introduce en la escotadura formada por los frontales (Figs. 1 y 2).

La serie infraorbital está constituída por 4 huesos (no se muestra en las figuras), carácter que concuerda con lo descrito por Springer (1993) para la familia, siendo el lacrimal el de mayor tamaño. Sostiene el canal sensorial que continúa hasta el esfenótico.

El vómer en vista ventral presenta una forma de «T» gruesa (Fig. 3), con una hilera de dientes cónicos y curvados posteriormente, mencionados por Regan (1912) para la familia y por Castillo \& Pequeño (1998) para el género. Este tipo de dientes se presenta también en todas las piezas óseas dentadas como palatino, premaxilares, dentarios y placas faríngeas; estos dientes son equivalentes a los de tipo "B" descritos por Casciotta \& Arratia (1993) para el suborden Labroidei.

La región orbital (Fig. 1, 2 y 3) está constituida por los huesos basisfenoides, proótico, pleurosfenoides y frontal. El basisfenoides presenta tres proyecciones laminares, dos laterales que articulan firmemente a la cara medial del proótico y un proceso sagital que se relaciona ventralmente con el parasfenoides. El pleurosfenoides (no se observa en 


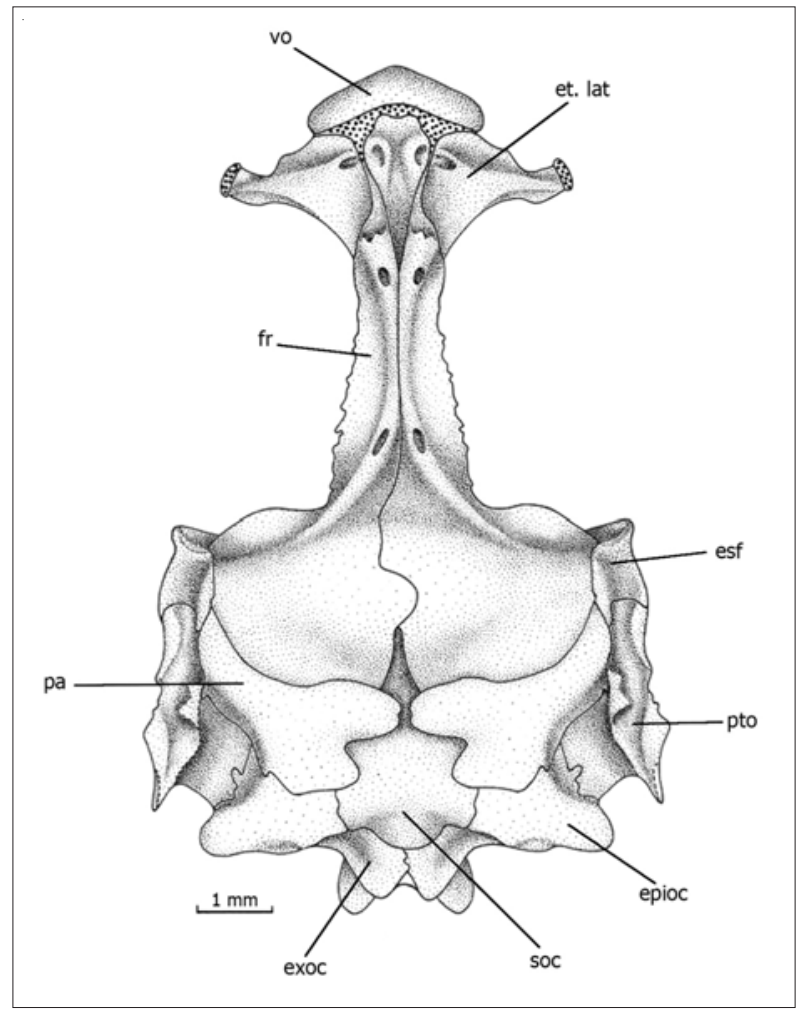

Figura 2. Vista dorsal del neurocráneo de H.chilensis. Fueron removidos del esquema los extraescapulares y postemporales / Dorsal view of the neurocranium of H.chilensis. The extrascapular and postemporal bones were removed

los esquemas debido a su posición sagital) articula dorsalmente a la cara medial del frontal y a la proyección medial del proótico.

El frontal presenta espinas simples desde los dos tercios posteriores del borde dorsal de la órbita ocular y en toda la superficie posterior; esta disposición de espinas es distinta a la observada en $H$. cunninghami, cuya disposición de las espinas se caracteriza por ser en duplas o tripletes (obs. pers. C. Cancino). Los frontales presentan una escotadura posterior y una sagital; los canales sensoriales supraorbitarios se contactan sagitalmente.

En la vista dorsal de la región ótica (Fig. 2) se muestra que los parietales se proyectan hacia la línea sagital, llegando incluso a contactarse en uno de los ejemplares estudiados. Se presenta un único extraescapular laminar, que se relaciona mediante tejido conectivo al parietal, pterótico y postemporal. En vista ventral (Fig. 3), se destaca por un lado que el proótico y el esfenótico forman típicamente la fosa para el cóndilo anterior del hiomandibular y por otra parte, que el foramen del nervio trigémino facial se encuentra en el borde anterior del

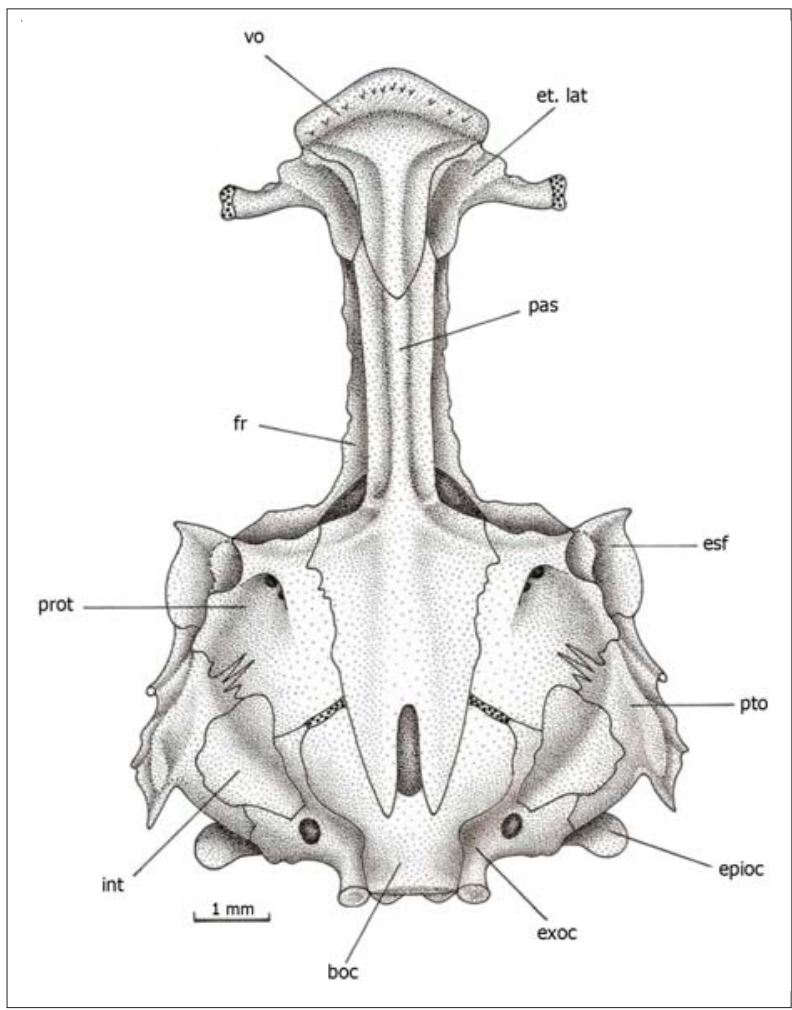

Figura 3. Vista ventral del neurocráneo de H.chilensis. No se representan los extraescapulares y postemporales / Ventral view of the neurocranium of $\mathrm{H}$. chilensis. The extrascapular and postemporal bones were removed

proótico, condición que ha sido descrita como carácter de Blennidae (Springer 1968) y que Cancino (1996) describe para las 4 especies de Scartichthys. La articulación proótico - pterótico es de tipo indentada, al igual que la observada en Calliclinus geniguttatus (obs. pers. S. Lampas).

El canal sensorial supratemporal está conformado por los huesos pterótico, esfenótico, extraescapular y preopercular. El intercalar es laminar, de diseño irregular y se sobrepone al proótico, exoccipital y pterótico (Fig. 1).

El parasfenoides forma la base sagital del neurocráneo (Fig. 3); la mitad anterior es más angosta, articula dorsalmente con el proótico y posteriormente con el basioccipital. El miódomo posterior está formado por el basioccipital y parasfenoides.

Tanto el basioccipital como el exoccipital alcanzan la misma distancia posterior. El basioccipital articula con el primer centro vertebral. El exoccipital muestra la escotadura posterior característica de perciformes, cuya proyección medial da forma a los cóndilos articulares para la primera vértebra. 


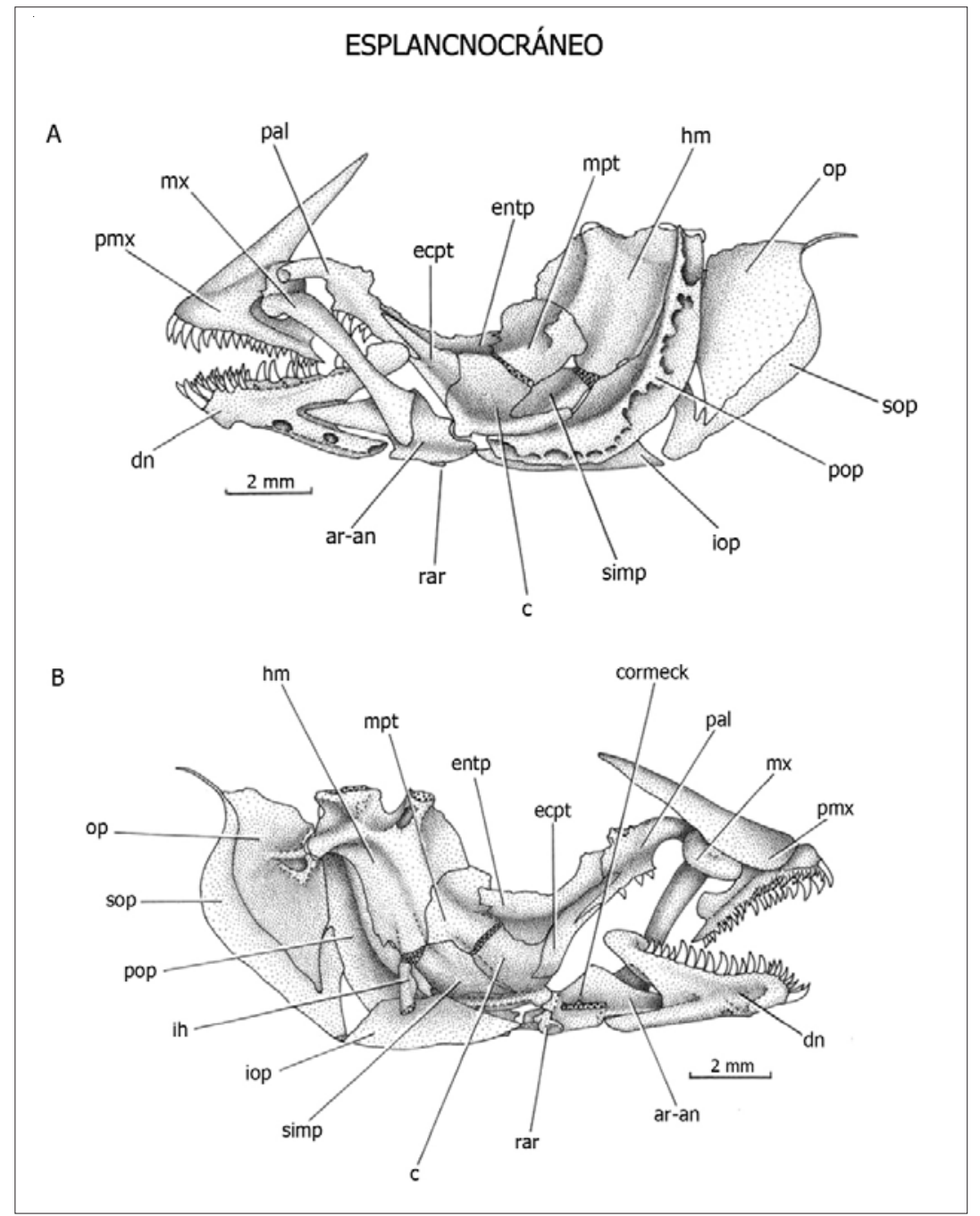

Figura 4. Vista lateral (A) y medial (B) del esplancnocráneo izquierdo de H.chilensis. (Se incluye el interhial) / Lateral (A) and internal (B) view of the left splanchnocranium of $\mathrm{H}$. chilensis (interhyal was included)

\section{ESPLANCNOCRÁNEO}

El esplancnocráneo se une posteriormente al neurocráneo mediante la articulación hiomandibular-proótico-esfenótico y anteriormente por medio de la articulación entre el palatino y el etmoides lateral.

Todos los huesos del suspensorio son planos, con bordes que articulan por solapamiento. El palatino, de diseño típico perciforme, posee un proceso anterior curvo que articula con el maxilar. Los huesos entopterigoides, metapterigoides, ectopterigoides y simpléctico son delgados, con poco tejido cartilaginoso entre ellos.

La serie opercular se observa estructuralmente desagregada, mientras que el opercular y subopercular se mantienen unidos y articulados al hiomandibular, el preopercular se une firmemente por su borde anterior al hiomandibular, simpléctico y cuadrado. Por su parte, el interopercular se adelanta para conectarse vía ligamento a la mandíbula inferior y medialmente es sostenido por el interhial, condición reportada para Scartichthys (Cancino, 1996).

El diseño de la mandíbula inferior y del premaxilar coinciden con lo descrito por Kotrschal (1988) para Tripterygiidae. Dentario y premaxilar sostienen una fila externa de dientes cónicos más robustos que aquellos dispuestos hacia el interior del extremo distal y sínfisis mandibular. El proceso ascendente del premaxilar es triangular y grande, lo que posibilita la protrusión premaxilar (Gon 1990, Castillo \& Pequeño 1998), 


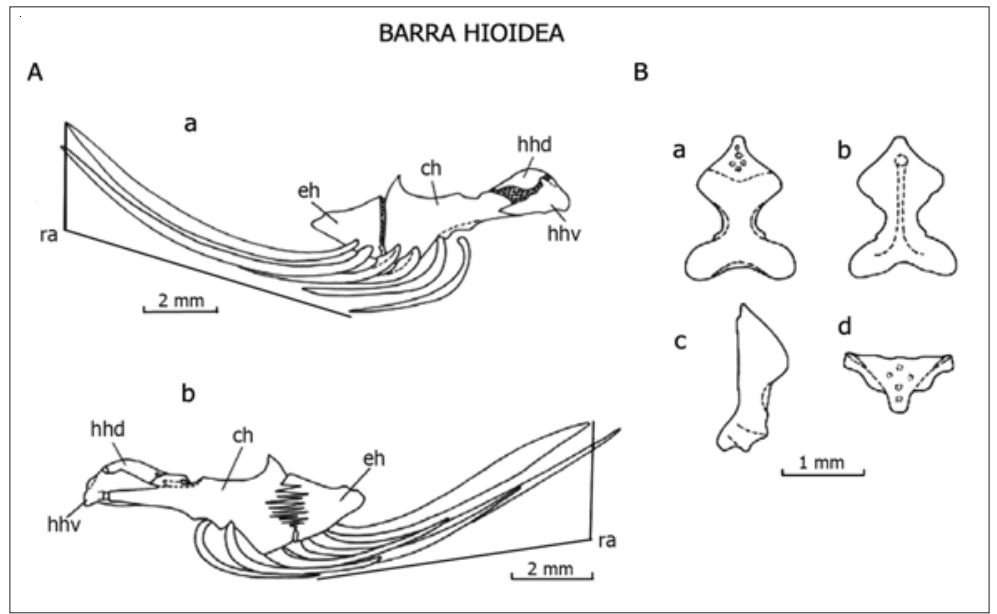

Figura 5. A. Vista lateral (a) y medial (b) de la barra hioídea izquierda de $\mathbf{H}$. chilensis. B. Detalle del urohial de $H$. chilensis en vista anterior (a), posterior (b), lateral (c) y ventral (d) / A. Lateral (a) and internal (b) views of the left hyoid bar of $\mathrm{H}$. chilensis. B. Anterior (a), posterior (b), lateral (c) and ventral (d) views of the urohyal of $\mathrm{H}$. chilensis característica descrita para la familia Tripterygiidae (Regan 1912, Springer 1993). El maxilar es plano y en su cabeza se presentan dos procesos: medial para el proceso ascendente del premaxilar y lateral para el palatino.

El cuadrado presenta gran desarrollo de su espina, lo que permite alojar gran parte del simpléctico; presenta un cóndilo ventral que lo relaciona al articular-angular. Están presentes el retroarticular y los cartílagos de Meckel y coronomeckeliano. El proceso anterior del articular-angular es triangular y se une al borde medial del dentario en una cavidad recubierta por tejido.

\section{Barra Hioidea}

Se ratifica el carácter presencia de 6 radios branquiostegales en Helcogrammoides (Castillo \& Pequeño, 1998), lo que coincide con el rango de 6 a 7 radios propuesto por Springer (1993) y Eschemeyer (2010) para Tripterygiidae. En tanto, Castillo \& Pequeño (1998) definen un número de seis radios branquiostegales para el género Helcogrammoides. Presencia de hipohiales unidos por tejido cartilaginoso, el ceratohial y el epihial con profunda articulación de tipo sinartrosis indentada. Cuatro radios articulan al ceratohial y los dos posteriores al epihial (Fig. 5A). El interhial de forma cilíndrica articula con la superficie lateral del epihial y sostiene la barra al esplancnocráneo, articulando con el hiomandibular (Fig. 4B).

Springer (1993) describe como característica de Blennidae la presencia de procesos laterales (dorsal y ventral) en el urohial, carácter que es corroborado por Cancino (1996) en Scartichthys. Sin embargo, en el mismo trabajo de Springer (1993) se menciona que es característica de Tripterygiidae la "ausencia” de dichos procesos. No obstante, en $H$. chilensis se observa un urohial con procesos laterales claramente definidos (Fig. 5B).

\section{Aparato Faringobranquial}

El basihial y basibranquiales 1 - 3 son cilíndricos, el cuarto basibranquial es romboídeo y cartilaginoso. El primer y segundo hipobranquial poseen procesos laterales laminares, mientras que el tercer hipobranquial presenta un proceso anterior cilíndrico. Los ceratobranquiales son largos y curvos y sostienen numerosas pequeñas branquispinas. Sólo el primer epibranquial es laminar, los restantes tres son cilíndricos y articulados a la única placa faríngea superior, que posee el mismo tipo de dentición que la placa faríngea inferior (Fig 6).

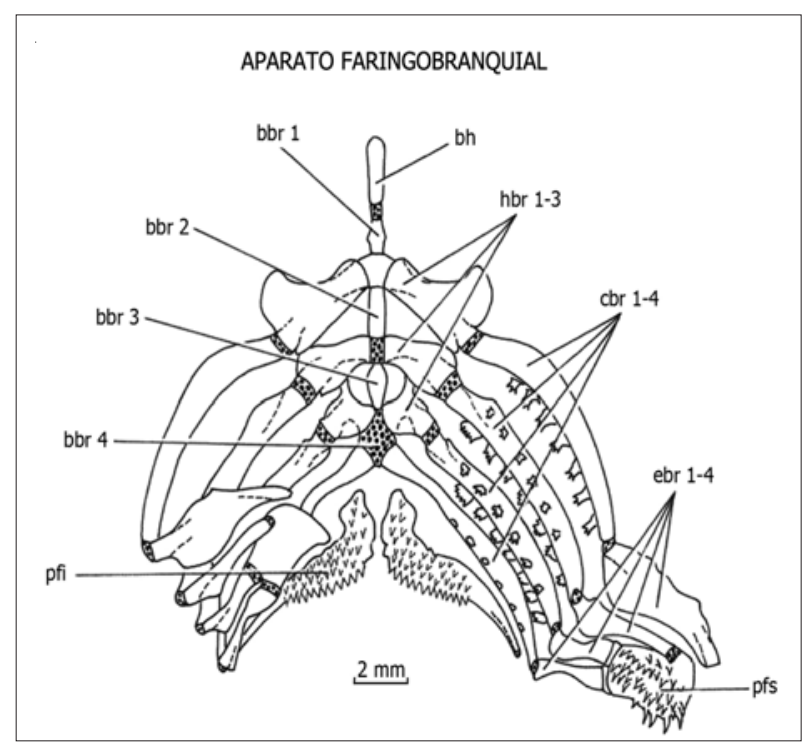

Figura 6. Vista dorsal del aparato faringobranquial de H.chilensis. Las branquispinas izquierdas no son representadas, los epibranquiales y placa faríngea superior del lado derecho se han extendido / Dorsal view of the pharyngobranchial apparatus of $\mathrm{H}$. chilensis. Left gill rakers were removed, epibranchials and right upper pharingeal plate folded out 


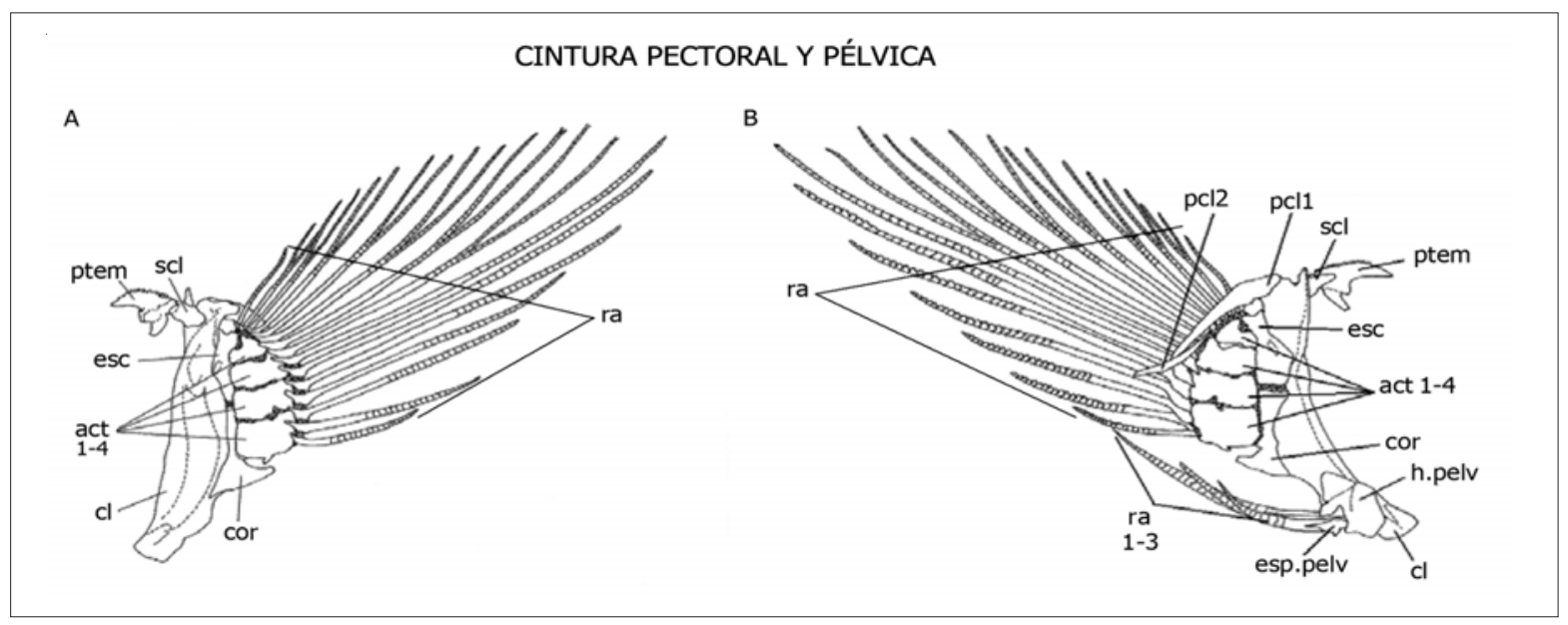

Figura 7. Vista lateral (A) de la cintura y aleta pectoral izquierda. Vista medial (B) muestra la relación entre la cintura pélvica y la cintura pectoral / Lateral view (A) of the left pectoral girdle and fin. The internal view (B) shows the relation between pelvic fin and pectoral girdle

\section{Cinturas Pectoral y Pélvica}

El postemporal está curvado dorsalmente, con espinas similares a las que sostiene el frontal. El cleitrum es amplio y unido medialmente al hueso pélvico y a dos postcleitrum. El coracoides es rectangular, con un proceso postcoracoideo laminar. Hay presencia de cuatro actinosteos que sostienen a los radios pectorales simples (entre 8 y 9) y ramificados (entre 7 y 8) (Castillo \& Pequeño 1998, Springer 1993). El foramen escapular está ubicado en la zona media anterior. (Fig. 7).

El margen anterior del hueso pélvico no sobrepasa el borde anterior del cleitrum, carácter descrito por Springer (1993) para Tripterygiidae. La aleta pélvica presenta de manera característica una espina y tres radios segmentados (Fig. 8).

\section{Aletas Anal y Dorsal}

El primer pterigióforo basal de la aleta anal se superpone con la primera espina hemal, mientras que los restantes se ubican entre espinas, manteniéndose una relación 1:1 entre pterigióforo y espina hemal (Fig. 9). La aleta dorsal es tripartita, observándose una clara diferenciación estructural entre la segunda y tercera aleta dorsal, debido a la presencia de un pterigóforo que no sostiene radio, lo que es considerado por Springer (1993) como exclusivo de Tripterygiidae. En forma característica, el primer pterigióforo de la primera dorsal sostiene dos radios y se ubica por delante de la espina neural, mientras que el segundo pterigióforo sostiene sólo un radio y se relaciona

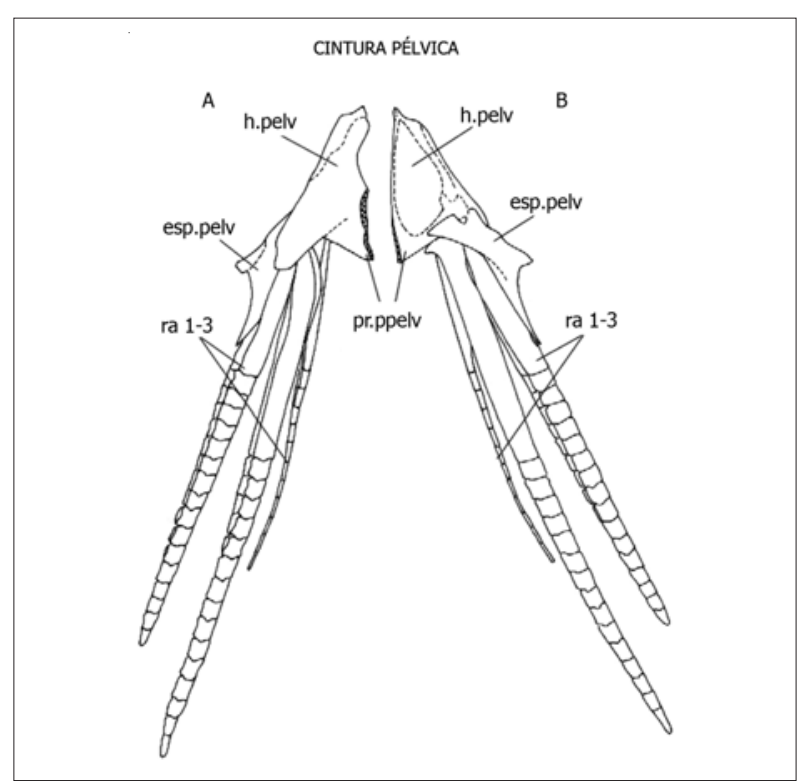

Figura 8. Vista dorsal (A) y ventral (B) de la cintura y aleta pélvica de $\mathbf{H}$. chilensis / Dorsal (A) and ventral (B) views of the left pelvic girdle and fin of $\mathrm{H}$. chilensis

con el segundo y tercer arcos neurales y con la espina neural de la cuarta vértebra.

Las relaciones anteriormente descritas para las aletas anal y dorsal, han sido definidas por Springer (1993) como caracteres para el suborden Blennioidei. Este mismo autor destaca que tanto en la aleta anal como en la dorsal, el último pterigióforo soporta dos radios. 


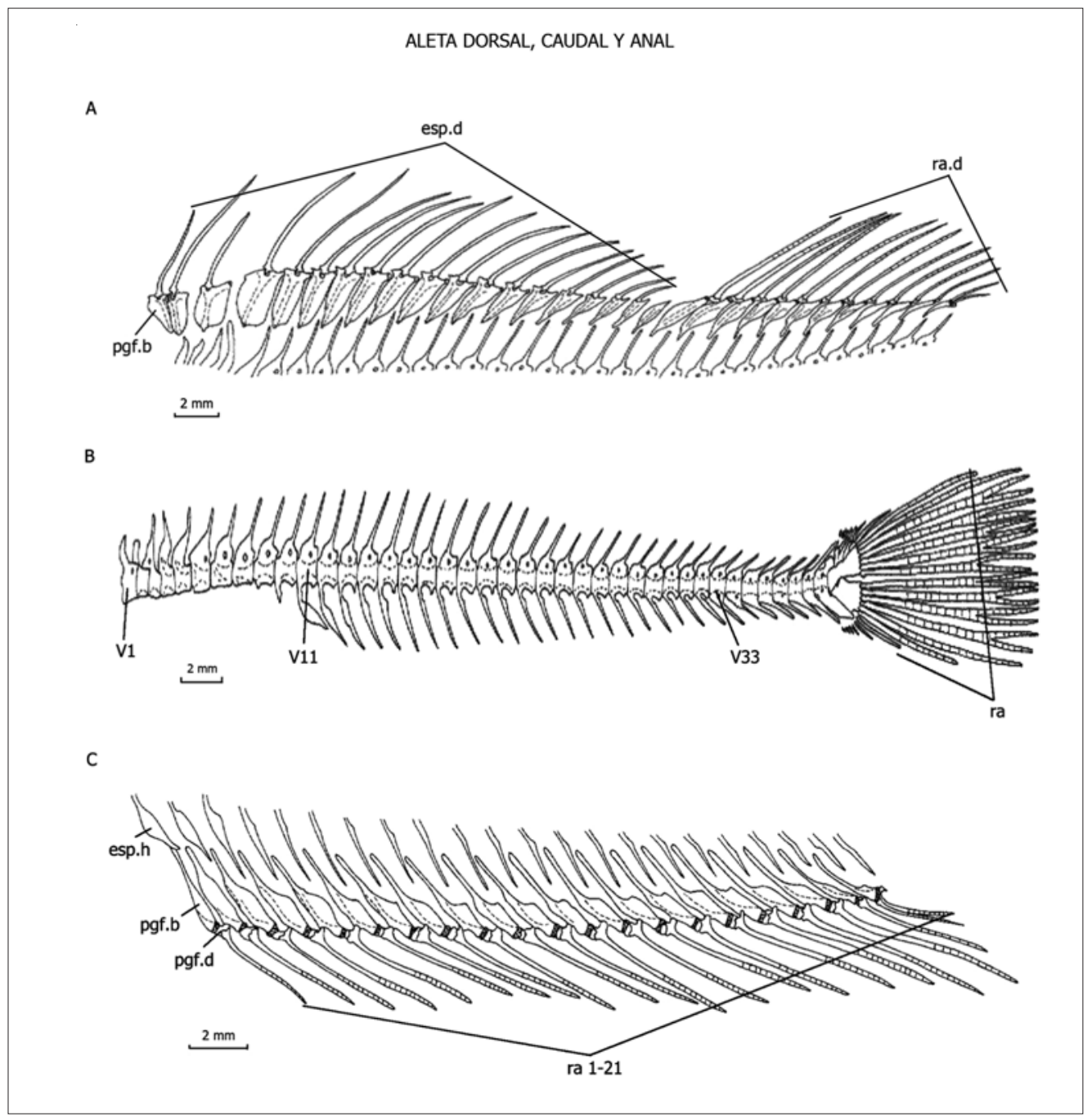

Figura 9. Vista lateral del lado izquierdo de A) aleta dorsal, B) columna vertebral, complejo uróforo y aleta caudal y C) aleta anal de $\mathbf{H}$. chilensis/ Lateral view of the left side of A) dorsal fin, B) vertebral column, urophore complex and caudal fin, and $\mathrm{C}$ ) anal fin of $\mathrm{H}$. chilensis

\section{Columna Vertebral}

En los especímenes analizados el conteo de cuerpos vertebrales fue de 41,10 precaudales y 31 caudales, encontrándose siempre que las primeras dos vértebras carecen de espina neural. La única excepción fue el especimen de 56,46 mm LS, en el que se contabilizó un total de 40 cuerpos vertebrales. El supraneural está ausente, lo que es un carácter que Springer (1993) establece para el suborden Blennioidei.

La primera espina hemal, la cual está curvada hacia la zona posterior, aparece entre las vértebras 9 y 11, posición que define la división entre las vértebras precaudales y caudales (Falabella et al. 1995). De las diez costillas epipleurales, las primeras dos se unen al arco neural de la primera y segunda vértebras, las restantes se unen al arco neural y a la región media de las costillas pleurales (no se muestran en los esquemas).

\section{Aleta Caudal y Complejo Uróforo}

La aleta caudal presenta una placa hipural dorsal que corresponde a la fusión del $3^{\circ}$ y $4^{\circ}$ hipural y una placa hipural ventral formada por la fusión del parihipural, hipurales 1 y 2. El quinto hipural se observa como una estructura independiente. Solo dos epurales articulan por separado a la placa hipural dorsal. Tanto el complejo preural II como el III presentan la espina hemal fusionada (Fig. 10). 


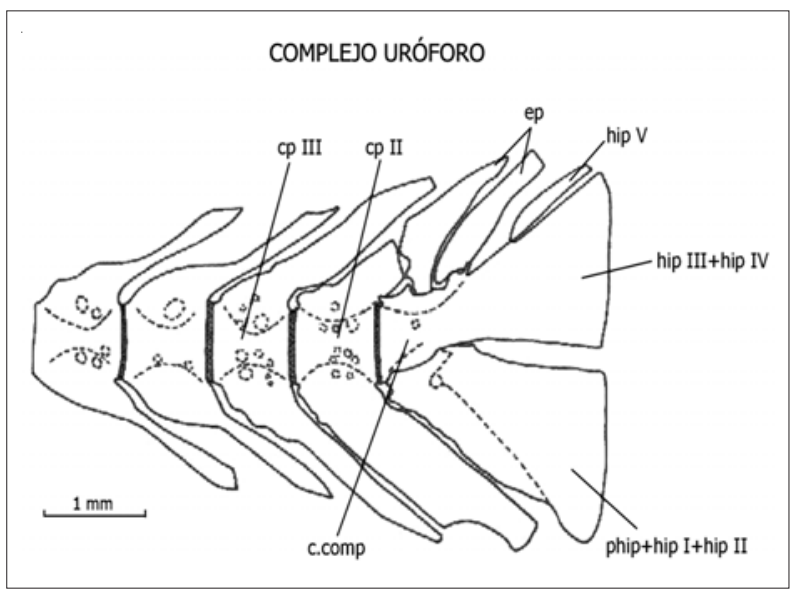

Figura 10. Vista lateral del lado izquierdo del esqueleto caudal de $\mathbf{H}$. chilensis, al cual le fueron removidos los radios principales y procurrentes / Left lateral view of the caudal skeleton of $\mathrm{H}$. chilensis. Principal and procurrent rays were removed

La organización del complejo uróforo es similar a la descrita por Clark (1979) para los géneros Norfolkia y Tripterygion, así como para Entomacodus nigricans Gill, 1859 y Parablennius parvicornis Valenciennnes, 1836, descritos por Springer (1968 y 1993, respectivamente).

\section{Conclusiones}

La ausencia de espina neural en las dos primeras vértebras, complejo uróforo altamente fusionado, urohial con procesos laterales desarrollados y la presencia de espina pélvica, son caracteres que contribuyen a la diagnosis de Helcogrammoides.

Neurocráneo con abundantes y pequeñas espinas dorsales. La escasez de tejido cartilaginoso y las articulaciones sinartrosis de la región posterior del neurocráneo, biseladas e indentadas, determinan una zona de alta resistencia mecánica, a la cual se articula el esplancnocráneo. Mientras que en la región anterior del neurocráneo el diseño de las estructuras determina una amplia órbita ocular que sostiene globos oculares laterales y sobresalientes. El espacio interorbital está reducido por el desarrollo del cartílago interorbital, que se presenta mayormente osificado, así como por la posición y desarrollo del basisfenoides.

La región etmovomeriana se proyecta anteroventralmente dado el diseño y relaciones espaciales que se establecen entre mesetmoides, etmoides laterales y vómer, lo que determina una superficie inclinada para el desplazamiento del proceso ascendente del premaxilar.

Esplancnocráneo, con estructuras bucales macizas y dientes cónicos. La serie opercular se observa desagregada, ya que el preopercular está firmemente articulado al suspensorio, mientras que el interopercular se adelanta para relacionarse con la mandíbula inferior, dejando a los huesos opercular y subopercular articulados entre sí y al hiomandibular.

La barra hioídea sostiene seis radios branquiostegales, los cuatro primeros articulados al ceratohial. Urohial con dos procesos laterales. De manera característica la barra es pequeña y es sostenida por el interhial que articula con el epihial.

Aparato faringobranquial achatado anteroposteriormente y expandido en forma lateral, sostiene una placa faríngea superior con pequeños dientes cónicos. Destaca el desarrollo diferenciado de los hipobranquiales.

El análisis morfológico de las estructuras óseas y superficies articulares de la cintura pectoral y aleta pectoral; cintura pélvica y aleta pélvica; así como el de las aletas impares dorsal, ventral y caudal, muestran un patrón de distribución estructural bastante constante, al igual que la longitud de los elementos óseos. Por lo que el diseño de los elementos constituyentes del tronco en Helcogrammoides chilensis, de la zona cental de Chile presenta baja variabilidad.

La descripción gráfica y explicativa de la osteología de complejos estructurales presenta como fortaleza la inclusión de dos niveles de análisis estructurales concatenados. Primero, a nivel estructural, es decir, el reconocimiento de la forma y el diseño de cada elemento óseo y segundo, a nivel de unidad funcional, el cual queda determinado por la agregación de los elementos que la constituyen, de tal manera que este nuevo diseño corresponde a una propiedad que emerge de la asociación entre las partes.

\section{Agradecimientos}

Las autoras agradecen al Dr. Eduardo de la Hoz (Q.E.P.D.), al Dr. Germán Pequeño por la donación de especímenes de Helcogrammoides. Al Dr. Roberto Meléndez C. y Dr. Bryan Dyer H. por su apoyo durante la elaboración de este artículo. A los evaluadores por sus valiosos comentarios y de manera muy especial al editor en jefe, Sr. Fernando Balbontín por su colaboración durante la revisión del manuscrito.

La Prof. Cecilia Cancino Araya dedica este trabajo a la memoria del Prof. Ismael Kong Urbina, ya que sobre la base de estos resultados se centraron nuestras últimas discusiones acerca de la importancia de la formación de 
jóvenes en ciencia; así como el valor de los estudios de carácter morfológico descriptivo en la resolución de los problemas en Taxonomía y Sistemática de Peces, durante la VII Reunión Bianual de Ictiología, Valdivia 2004.

\section{LITERATURA CITADA}

Barel CDN. 1983. Towards a constructional morphology of cichlid fishes (Teleostei, Perciformes). Netherlands Journal Zoology 33(4): 357-424.

Bock M \& CD Zander. 1986. Osteological characters as tool for Blennid taxonomy, a generic revision of European Blenniidae (Percomorphi; Pisces). Sonderdruck aus Zeitschrift für Zoologische Systematik und Evolutionsforshung Band 24(2): 138-143.

Bussing WA. 1991. A new genus and two new species of tripterygiid fishes from Costa Rica. Revista de Biología Tropical 39(1): 77-85.

Cancino C. 1955. Peces de las pozas litorales de Chile Central. Memoria de Profesor de Biología y Química, Facultad de Filosofía y Educación, Universidad de Chile, Santiago, 135 pp.

Cancino C. 1996. Diseño estructural cefálico y mecanismos de captura de alimento de Scartichthys (Perciformes, Blenniidae): adaptaciones hacia herbivoría. Tesis de Magíster en Ciencias Biológicas, con mención en Ecología y Sistemática, Pontificia Universidad Católica de Valparaíso, 108 pp.

Cancino C, E de la Hoz \& K Farías. 1999. Morfología cefálica de Hypsoblennius sordidus (Bennet, 1828) (Perciformes, Blenniidae): un blénido omnívoro. Revista de Biología Marina y Oceanografía 34(2): 167-180.

Casciotta JA \& G Arratia. 1993. Jaws and teeth of American Cichlids (Pisces: Labroidei). Journal of Morphology 217: 136.

Castillo M \& G Pequeño. 1998. Sinopsis de Tripterygiidae de Chile (Osteichthyes: Perciformes). Gayana Zoologia 62(2): 109-133.

Clark E. 1979. Red Sea fishes of the Family Tripterygiidae with descriptions of eight species. Israel Journal of Zoology 28: 65-113.

Dingerkus G \& K Uhler. 1977. Enzyme clearing of alcian blue stained whole small vertebrate for demostration of cartilage. Stain Technology 52: 229-232.

Dullemeijer P. 1974. Concepts and approaches in animal morphology, 264 pp. Van Gorkum, Assen.

Dullemeijer P \& CDN Barel. 1977. Functional morphology and evolutionary biology. Acta Biotheoretical 29: 151-250.

Eschmeyer W. 2010. Catalog of fishes. California Academy of Science. [en línea] <http://www.calacademy.org/research/ ichthyology/catalog/fishcatsearch.html>

Falabella F, R Meléndez \& L Vargas. 1995. Claves osteológicas para peces de Chile central: un enfoque arqueológico, 208 pp. Editorial Artegrama, Santiago.
Fricke R. 1991. Ceratobregma striata, a New Triplefin (Tripterygiidae) from Northern Australia, and Record of Norfolkia brachylepis from Western Australia. Japanese Journal of Ichthyology 37(4): 337-343.

Gon O. 1990. Synaphobranchidae. En: Gon O \& PC Heemstra (eds). Fishes of the Southern Ocean, pp. 102-104. JLB Smith Institute of Ichthyology, Grahamstown.

Hadley PE. 1986. Revision of the Tripterygiid fish genus Helcogramma, including descriptions of four new species. Bulletin of Marine Science 38(2): 313-354.

Kotrschal K. 1988. A catalogue of skulls and jaws of easterns tropical pacific blennioid fishes (Blennioidei: Teleostei): a proposed evolutionary sequence of morphological change. Journal of Zoological Systematics and Evolutionary Research 26: 442-466.

Lauder GV \& KF Liem. 1983. The evolution and Interrelationships of the Actinopterygian Fishes. Bulletin of the Museum of Comparative Zoology 150(3): 95-197.

Liem KF. 1970. Comparative functional anatomy of the Nandidae (Pisces: Teleostei). Fieldiana Zoology 56: 1-166.

Quast JC. 1965. Osteological characteristics and affinities of the hexagrammid fihes, with a synopsis. Proceedings California Academy of Sciences 31(21): 563-600.

Regan CT. 1912. The classification of the Blennioid fishes. Annals and Magazine of Natural History 57: 265-280.

Rosenblatt RH. 1959. A revisionary study of the blennioid fish family Tripterygiidae. Ph. D. Thesis, University of California, Los Angeles, 376 pp.

Rosenblatt RH. 1960. The atlantic species of the Blennioid fish genus Enneanectes. Proceedings of the Academy of Natural Sciences of Philadelphia 112: 1-23.

Springer VG. 1968. Osteology and classification of the fishes of the Family Blenniidae. United States National Museum Bulletin 284: 1-85.

Springer VG. 1993. Definition of the Suborder Blennioidei and its included families (Pisces: Perciformes). Bulletin of Marine Science 52(1):472-495.

Starks EC. 1926. Bones of the ethmoid region of the fish skull. Stanford University Publication, Biological Sciences 4(3): 139-338.

Tomo AP. 1981. Contribución al conocimiento de la fauna ictiológica del sector Antártico Argentino. Publicacion. Instituto Antártico Argentino 14: 1-242.

Weitzman SH. 1962. The osteology of Brycon meeki, a generalized characid fish, with an osteological definition of the family. Stanford Ichthyological Bulletin 8(1): 1-77.

Wheeler A \& J Dunne. 1975. Tripterygion atlanticus sp. nov. (Teleostei - Triperygiidae) the first record of a tripterygiid fish in Norh-Western Europe. Journal of Fish Biology 7: 639-649.

Wiley EO. 1981. Phylogenetics. The theory and practice of phylogenetics systematics, 456 pp. John Wiley \& Sons, New York.

Recibido el 11 de agosto de 2009 y aceptado el 29 de abril de 2010 
Anexo 1. Lista de Abreviaturas / Abbreviation list

\begin{tabular}{|c|c|}
\hline act $1-4$ & : actinósteo 1-4 \\
\hline ar-an & : articular- angular \\
\hline bbr 1-4 & : basibranquiales 1-4 \\
\hline bh & : basihial \\
\hline boc & : basioccipital \\
\hline bsf & : basisfenoides \\
\hline c & : cuadrado \\
\hline car.iorb & : cartílago interorbital \\
\hline cbr 1-4 & : ceratobranquiales 1-4 \\
\hline $\mathrm{cl}$ & : cleitrum \\
\hline c.comp & : centro compuesto \\
\hline cor & : coracoides \\
\hline cormeck & : coronomeckeliano \\
\hline cpII & : centro pleural II \\
\hline ср III & : centro pleural III \\
\hline ch & : ceratohial \\
\hline $\mathrm{dn}$ & : dentario \\
\hline ebr $1-4$ & : epibranquiales 1-4 \\
\hline ecpt & : ectopterigoides \\
\hline eh & : epihial \\
\hline entp & : entopterigoides \\
\hline ep & : epural \\
\hline еріoc & : epioccipital \\
\hline esc & : escapular \\
\hline esf & : esfenótico \\
\hline esp. d & : espina dorsal \\
\hline esp. h V 16 & : espina hemal \\
\hline esp. pelv & : espina pélvica \\
\hline et. lat & : etmoides lateral \\
\hline exoc & : exoccipital \\
\hline $\mathrm{fr}$ & : frontal \\
\hline h. pelv & : hueso pélvico \\
\hline hbr 1-4 & : hipobranquial 1-4 \\
\hline hhd & : hipohial dorsal \\
\hline hhv & : hipohial ventral \\
\hline
\end{tabular}

\begin{tabular}{|c|c|}
\hline hip III + hip IV & : tercer hipural más cuarto hipural \\
\hline hipV & : quinto hipural \\
\hline hm & : hiomandibular \\
\hline ih & : interhial \\
\hline int & : intercalar \\
\hline iop & : interopercular \\
\hline meset & : mesetmoides \\
\hline mpt & : metaperigoides \\
\hline $\mathrm{mx}$ & : maxilar \\
\hline op & : opercular \\
\hline pa & : parietal \\
\hline pal & : palatino \\
\hline pas & : parasfenoides \\
\hline pcl 1 & : poscleitrum 1 \\
\hline pcl 2 & : poscleitrum 2 \\
\hline pfi & : placa faríngea inferior \\
\hline pfs & : placa faríngea superior \\
\hline pgf.b & : pterigióforo basal \\
\hline pgf. d & : pterigióforo distal \\
\hline phip+ hipI+ hipII & I : parhipural + hipural I + hipural II \\
\hline pmx & : premaxilar \\
\hline pop & : preopercular \\
\hline prot & : proótico \\
\hline pr.ppelv & : proceso postpélvico \\
\hline ptem & : postemporal \\
\hline pto & : pterótico \\
\hline ra & : radios \\
\hline ra $1-21$ & : radios $1-21$ \\
\hline ra $1-3$ & : radios pélvicos 1-3 \\
\hline ra.d & : radios aleta dorsal \\
\hline rar & : retroarticular \\
\hline scl & : supracleitrum \\
\hline $\operatorname{simp}$ & : simpléctico \\
\hline soc & : supraoccipital \\
\hline sop & : subopercular \\
\hline v 1, 11, 33 & : vértebras 1 , 11, 33 \\
\hline vo & : vómer \\
\hline
\end{tabular}

